EOMmun Communication et organisation



Âges et générations : la communication revisite ses publics

\title{
Le marché des jeux vidéo
}

Univers de substitution et fabrique de l'opinion

Laurent Trémel

\section{CpenEdition}

Journals

Édition électronique

URL : http://journals.openedition.org/communicationorganisation/3563

DOI : 10.4000/communicationorganisation.3563

ISSN : 1775-3546

Éditeur

Presses universitaires de Bordeaux

Édition imprimée

Date de publication : 1 décembre 2011

Pagination : 103-112

ISBN : 978-2-86781-745-8

ISSN : 1168-5549

\section{Référence électronique}

Laurent Trémel, «Le marché des jeux vidéo », Communication et organisation [En ligne], 40 | 2011, mis en ligne le 01 décembre 2014, consulté le 01 mai 2019. URL : http://journals.openedition.org/ communicationorganisation/3563 ; DOI : 10.4000/communicationorganisation.3563 


\title{
Le marché des jeux vidéo Univers de substitution et fabrique de l'opinion
}

\author{
Lourent Trémel ${ }^{1}$
}

Depuis une vingtaine d'années, l'industrie du jeu vidéo a pris une place prépondérante dans le domaine des loisirs des jeunes, au point que certains indicateurs montrent que le chiffre d'affaires de ce marché serait aujourd'hui supérieur à celui du cinéma (hors produits dérivés).

Le propos de cet article est de cerner les stratégies de marketing, et de lobbying, de l'industrie des jeux vidéo visant à la fois à fournir des univers de substitution, de plus en plus " captivants ", pour les publics prioritairement intéressés par ces logiciels (les adolescents et les jeunes adultes de sexe masculin), tout en tentant d'atteindre de nouvelles « cibles " (femmes, cadres, retraités, etc.) par la mise en avant de "bons produits » s'éloignant des stéréotypes associés aux jeux vidéo et par la production de discours sur les « joueurs » destinés à faire l'opinion, pour reprendre une expression de Patrick Champagne (1990).

\section{Les mondes des jeux vidéo : culte du surhomme et compensation sociale}

Des chercheurs, réunis autour de l'universitaire canadien Stephen Kline (2003), ont bien défini ce qui faisait l'essence de la production vidéo-ludique : la masculinité militarisée. En termes quantitatifs (heures passées à jouer, fréquences de jeu, nature de l'offre de logiciels), les titres les plus joués, notamment sur consoles, sont des jeux d'action, de guerre, de combats, de sport, prônant des valeurs hyper-compétitives. Au sein de ces univers, les joueurs incarnent des magiciens et des guerriers puissants, évoluant dans des univers d'heroic-fantasy, fréquemment dotés de pouvoirs et d'une nature surbumaine, des chefs d'État et des généraux gérant des peuples et des armées tout dévoués à leur cause, ou encore des sportifs d'exception, se distinguant là aussi du « commun».

1. Laurent Tremel est Docteur en Sociologie de l'EHESS, Chargé de conservation et de recherche au Musée national de l'Education (CNDP, Rouen) ; laurent.tremel@cndp.fr 
En septembre 2010, dans le cadre des travaux de l'Université d'été de 1'Union Nationale des Clubs Universitaires ${ }^{2}$, nous avions réalisé une étude sur ces jeux vidéo de "sport » qui permettait de cerner très nettement d'une part, quelles étaient les « valeurs » mises en exergue dans ces jeux, et, d'autre part, à quelles fins elles étaient distillées dans ces univers de fiction.

Sur le site de l'éditeur Electronic Arts $^{3}$, se positionnant sur le marché des jeux vidéo comme l'un des principaux producteurs de jeux de sport, on pouvait ainsi lire en guise de préambule (paragraphe de présentation de la gamme) : "Grâce aux jeux de sport, vous incarnez enfin ce sportif de haut niveau qui participe aux plus grandes compétitions internationales dans un contexte de féroce concurrence. »

D'emblée, on remarquait ce qui était mis ici en exergue : grâce à ces jeux, le jeune pouvait incarner « enfin » $(s i c)$ un sportif de « haut niveau ». Il pouvait participer virtuellement « aux plus grandes compétitions internationales » dans un contexte de « féroce concurrence ». On comprenait aisément le public visé prioritairement : des jeunes de sexe masculin, et les dimensions de leur ego qui étaient sollicitées. Lidée de " féroce concurrence » était reprise quelques lignes plus tard par la référence « à des terrains où la compétition fait rage ».

On notait également dans le texte une utilisation abondante de superlatifs et d'expressions valorisantes : "références ", "franchises incontournables ", "EA sports est fier ", " expérience authentique », " titres les plus célèbres », " les plus beaux parcours de golf ", " vous menez votre équipe favorite à la gloire ", "formations surentraînées ", " entrée gagnante ", " incarner votre superstar NBA ».

De façon là encore explicite, le dernier paragraphe de cette présentation, intitulé «Devenez le meilleur joueur au monde », flattait l'ego du joueur, en mettant même en avant, significativement, la notion de "fantasme » : «Nos jeux de sport vous offrent de l'action plus vraie que nature pour que vous puissiez assouvir vos fantasmes sportifs les plus incroyables. La plupart de nos titres vous permettent de poursuivre l'expérience en ligne et de vous frotter aux meilleurs joueurs du monde. »

Cet exemple nous semble particulièrement significatif des logiques analysées au cours de nos travaux sur la pratique des jeux de rôles et des jeux vidéo, depuis le début des années 1990, et dont les principaux éléments ont été publiés dans un ouvrage faisant suite à notre soutenance de thèse (Trémel 2001) et dans d'autres publications plus récentes. Alors que, depuis une vingtaine d'années, les processus de mobilité sociale ascendante entre les générations sont remis en cause, que les jeunes sont confrontés massivement à la déqualification professionnelle au sortir de leurs études et à la précarisation

2. L. TRÉMEL, Virtualité et sport. Ecrans multiples, vidéo et cybersport, Communication lors de la $28^{e}$ Université sportive d'été organisée par l'Union Nationale des Clubs Universitaires, CREPS PACA, Aix-en-Provence, 9 septembre 2010.

3. Site d’Electronic Arts, [En ligne], http://www.ea.com/fr/genre/jeux-sport. 
des emplois auxquels ils peuvent prétendre, voire au chômage, se sont développés en parallèle des "univers virtuels » où ils peuvent acquérir des " grandeurs " via l'incarnation d'un avatar prédisposé à connaître un destin hors du commun. Le tout au sein de scénarios qui font la part belle à des valeurs néo-libérales.

Les éléments figurant sur le site d'Electronic Arts attestent d'une prise en compte explicite par les industriels du loisir des manques rencontrés dans la vie de tous les jours par les joueurs, afin de leur fournir des formes de compensation symboliques au sein des « terrains de jeu » ainsi créés.

Ces mécanismes potentiellement aliénants, et plus généralement, les thématiques développées dans les jeux vidéo les plus pratiqués, entraînent épisodiquement des critiques. On note ainsi principalement une critique de la "violence ", il est vrai complaisamment scénarisée dans certains jeux (renforcée avec les modélisations en 3D qui rendent les combats et l'élimination des adversaires plus " réalistes »). Mais aussi une dénonciation du machisme caractéristique de la mise en scène des rôles masculins et féminins dans ces " mondes $»^{4}$, ou encore parfois, une critique des valeurs néo-libérales et de l'individualisme prônés dans bon nombre de titres. Une psychologie accusatrice, fréquemment renforcée par des attaques politiques provenant d'associations de parents situées à droite de l'échiquier politique et plus rarement, de critiques provenant des " forces de gauche $»^{5}$, contribue à diffuser auprès de l'opinion une image trouble du monde des jeux vidéo.

Depuis plusieurs années, les industriels du loisir à l'origine de la production de jeux vidéo tentent donc, avec un certain succès, de modifier cette image par divers procédés. Le premier se rapporte à la définition du « joueur ». Ici, l'objectif est double : faire croire que le joueur de jeu vidéo n'est plus « un ado mordu par le jeu ", mais un citoyen responsable, consommant ces produits parmi d'autres biens culturels. Il s'agit alors de contrer les critiques provenant des milieux éducatifs et d'associations de parents, mais également de diffuser des représentations des joueurs susceptibles de permettre l'identification de publics, jusqu'alors peu concernés par la pratique (les femmes, et plus encore les retraités). La tentative d'amener à la pratique et donc à l'achat, des publics «solvables ", bénéficiant d'un fort pouvoir d'achat (cadres, retraités), potentiellement moins concernés par des velléités de piratage des produits (important chez les adolescents), apparaît en outre comme une nécessité économique afin de trouver de nouveaux débouchés à un marché, certes,

4. M. BALZERANI, "La femme n'est pas l'avenir du jeu vidéo », MCD, 2011, n64; F. LIGNON, " L'image de la femme dans les jeux vidéo de combat », dans ROGER A., TERRET TH. (dir.), Sport et Genre. XIXème-XXème, Paris, L'Harmattan, 2005, vol. 4, p. 171-185 ; F. LIGNON, " Genre et jeux vidéo », dans BAURENS M., JANNAS F., LIGNON F. (dir.), Le genre en éducation, Grenoble, éditions Eaux Claires, 2007, p. 97-103.

5. En sachant que dès lors que les jeux vidéo font l'objet d'attaques de la part de la droite " extrême ", certains intervenants se situant à gauche ont tendance à se présenter comme des avocats inconditionnels du médium, épousant eux aussi les discours des industriels sur certains points (par exemple l'analyse au "second degré » d'éléments affligeants développés dans certains titres). 
en pleine expansion, mais marqué par une forte concurrence et des crises épisodiques ${ }^{6}$.

Si le processus de diversification des publics du jeu vidéo est bien présent, aboutissant notamment aux phénomènes de "casual gaming " (jeu occasionnel), il sera en quelque sorte hypertrophié par les promoteurs de ces discours afin de "faire l'opinion » et donc de modifier l'image sociale des jeux vidéo. Ceci pourrait être résumé par le slogan : « des jeux adultes pour un public adulte ». En parallèle, les fabricants tenteront, via la publicité des chaines télévisées " généralistes ${ }^{7}$ aux heures de grandes écoute, et les liens qu'ils ont tissés avec des journalistes, de mettre en lumière une « vitrine présentable » de « bons produits » (non-violents, faisant appel à la " réflexion ", basés sur les images chatoyantes des dessins animés pour enfants, associant toute la famille dans des moments conviviaux, se référant à « l'histoire " ${ }^{8}$, etc.), présentant des dimensions culturellement légitimes. Depuis quelques mois, cette tendance est accentuée par la mise en avant des "serious games", dont la diffusion reste marginale en termes de marché, mais qui s'attirent les faveurs de toutes sortes d'observateurs technophiles vantant leurs mérites, les présentant comme d'excellents outils pédagogiques, des supports didactiques novateurs et efficaces.

\section{Du « vieillissement du joueur » au goldfarming : conséquences des évolutions de la pratique}

Même si peu de données fiables circulent actuellement, d'un point de vue sociologique, sur la pratique des jeux vidéo ${ }^{9}$, des indicateurs tendent à démontrer, au sein même des " enquêtes " dont nous critiquons les orientations, qu'en termes de fréquence et de durée de jeu, ce sont essentiellement les enfants, les adolescents et certains « jeunes adultes », de sexe masculin, qui sont concernés par ce phénomène. Dès lors, comment expliquer que les éditeurs de jeux, les médias, voire certains "savants ", avancent aujourd'hui que la moyenne d'âge $\mathrm{du}$ « joueur de jeux vidéo » serait comprise entre 30 et 39 ans $^{10}$ ?

6. L'industrie du jeu vidéo française connaît ainsi une situation de crise endémique depuis le début des années 2000, ayant conduit au développement d'un fort taux de chômage dans cette branche.

7. Notons d'ailleurs que celles-ci contrastent avec les publicités destinées aux " gamers ", plutôt diffusées en seconde partie de soirée et sur les chaînes de la TNT prisées par les adolescents (NRJ 12, NT1, Direct Star, W9). Celles-ci assurent la promotion de jeux vidéo plus « traditionnels » comme les jeux de guerre et de combats, ou encore les jeux de sport.

8. Citons en ce sens la série des jeux Civilization, valorisée par plusieurs observateurs et des pédagogues technophiles, développant des postulats pour le moins ethnocentrés, ayant fait l'objet de travaux d'analyse critique menés avec la collaboration de Tony Fortin.

9. Le CREDOC devrait toutefois réaliser dans les prochains mois une enquête d'ampleur sur la question. 10. Récemment, on peut se reporter à l'étude réalisée en 2010 par la Fédération européenne des logiciels de loisirs (ISFE), mettant entre autre en avant le fait que 38\% des Français seraient considérés comme " joueurs » (puisqu'ayant utilisé un jeu vidéo lors des six derniers mois...) ou encore que l'âge moyen des pratiquants serait de 35 ans alors que l'on compterait parmi eux une moitié de femmes... 
Nous avons eu l'occasion d'étudier plus précisément la résultante de ce processus de lobbying (reprise de ces statistiques) dans plusieurs publications, auxquelles on peut se reporter ${ }^{11}$. Il s'agit notamment de la presse "populaire " (ex. le magazine Carrefour savoir, distribué dans cette chaîne de supermarchés), la presse « légitime » (ex. articles publiés dans $L e$ Monde ${ }^{12}$ ), et certains travaux universitaires. Autre exemple récent, et particulièrement problématique de cette tendance, dans la revue professionnelle Le Journal des psychologues ( ${ }^{\circ} 291$, octobre 2011), une psychologue clinicienne qualifiée de « spécialiste des nouvelles technologies et des addictions » reprend mécaniquement, sans les interroger le moins du monde, les données et les raisonnements de l'industrie du jeu vidéo sur « la démocratisation des jeux » et les « nouveaux joueurs ». Sûre de ces statistiques, elle en vient à rejeter l'image du joueur ado jouant " excessivement », et celle portant sur les " jeux de combats sur console ou ordinateur », vers le registre de «l'inconscient collectif» (sic) ou encore du « cliché »...

On le comprend bien, la " moyenne d'âge » du joueur à 30 ans (et plus) a pour avantage, du point de vue des fabricants de jeux et des lobbyistes, de permettre de brandir cet argument contre les associations familiales qui s'inquiètent du contenu discutable de certains jeux vidéo et pensent que ces produits concernent des « enfants »... Mais il s'agit là d'un artefact statistique.

$\mathrm{Au}$ niveau mathématique, de telles " moyennes " sont souvent obtenues en "sondant " des panels de répondants âgés de 16 ans et plus, excluant donc d'emblée les plus jeunes qui composent pourtant les plus gros bataillons des aficionados. Les critères retenus pour identifier les « joueurs" sont peu discriminants : au Canada, l'Entertainment Software Association fixe ainsi la moyenne d'âge du « joueur » à 39 ans (!), mais il faut savoir que l'on " ratisse large », puisqu' est incluse dans cette catégorie « toute personne ayant au moins joué à un jeu vidéo une fois dans l'année »...

Dans d'autres enquêtes ${ }^{13}$, où l'on affirme cette fois-ci s'intéresser aux pratiques des "plus jeunes ", ce sont en fait toujours des répondants de plus de 16 ans qui sont sondés, mais on leur demande de décrire comment les autres membres du foyer « jouent aux jeux vidéo ». En pratique, ce sont donc fréquemment les parents qui sont amenés à " évaluer " la façon dont leurs enfants jouent. Or, ce ne sont pas forcément les mieux placés, notamment lorsqu'il s'agit de savoir précisément ce que font des adolescents dont chacun sait qu'ils peuvent être tentés de dissimuler certains aspects de leurs activités aux adultes...

11. L. TRÉMEL, "Des jeux vidéo à la "réalité virtuelle" : réflexions sur un objet d'études aux prises avec la réalité sociale » dans TRÉMEL L. (dir.), Les pratiques audiovisuelles. Réflexions sur des questions d'éducation, de culture et de consommation de masse, Dijon, Éditions d'un Autre genre, 2009.

12. S. CHEMLA «Typologie des joueurs », Le Monde, 10 décembre 2004.

13. Enquête TNS-SOFRES, octobre 2006. 
L'ensemble, fait de « moyennes " qui n'ont pas grand sens d'un point de vue sociologique (car elles masquent des usages très contrastés), conduit à diffuser auprès de la population une image biaisée, mais présentant des aspects « rassurants » de la pratique vidéoludique : la « moyenne d'âge » est élevée et des données telles que la fréquence de jeu ou encore la durée de jeu hebdomadaire sont minorées du fait de la prise en compte du casual gaming.

De même, on obtient également une proportion élevée de " joueuses » (de l'ordre de $40 \%$ à $50 \% . .$.$) pouvant laisser croire que les jeux vidéo ne seraient$ pas si «machistes » que certains le prétendent et que des jeunes filles (telles que celles au physique avenant mises en scène dans les publicités télévisées et qui semblent s'intéresser à des jeux pour adolescentes dont elles singent le comportement) voire des femmes d'âge mûr, seraient aujourd'hui des aficionadas de ces loisirs numériques.

Dans cette construction statistique, un cadre jouant occasionnellement sur son PC entre deux rendez-vous, ou encore une mère de famille pratiquant un jeu de cartes sur le système d'exploitation de l'ordinateur aura le même « poids » qu'un adolescent déscolarisé jouant 10 heures par jour. Les quelques minutes passées quotidiennement par ces personnes à jouer (voire des fréquences encore plus irrégulières comme une ou deux fois par semaine par exemple), feront littéralement " fondre » les moyennes des "gamers ». De même, au niveau des "genres de jeu » les plus pratiqués, on parvient ainsi à une surévaluation des produits pour $\mathrm{PC}$, à une surévaluation de genres de jeux et/ou de titres "politiquement corrects ", et à une minoration de la part des jeux de combat, de tir ou de guerre sur consoles.

Or, il convient de rappeler qu'en fait, l'essentiel du marché des jeux vidéo concerne des jeux pour consoles de salon (60\%) et consoles portables, type «game boy» (25\%). Les logiciels pour PC (parmi lesquels on va trouver les produits les plus « culturels » dont certains observateurs vantent les mérites) ne représentent que $15 \%$ de l'ensemble ${ }^{14}$.

Sur console, environ $75 \%$ des jeux pratiqués consistent en des jeux de guerre et de combats répétitifs, des jeux dits d'action et d'autres de « sport » qui mettent en scène des sortes de dieux du stade. Au sein de ces produits, notons la violence présente dans de nombreux titres, le machisme distillé dans les scénarios (outre le militarisme, on peut ainsi opposer l'aspect « viril » du sport de compétition à un "sport féminin " très souvent associé ici à la gym et au fitness...), et des idéologies très en phase avec la pensée politique des néo-conservateurs américains (notamment en matière de lutte contre le terrorisme $)^{15}$.

On pourrait aussi évoquer le procédé utilisé par les industriels du secteur sur certains plateaux de télévision ou de radio, alors qu'ils sont opposés à des

14. L. TRÉMEL, T. FORTIN, Mythologie des jeux vidéo, Paris, Le Cavalier bleu, "collection Mythô ", 2009, p. 22-23.

15. Ibid., p. 21-38. 
parents hostiles à ces loisirs. Benoîtement, ils leur expliquent que seule une minorité des jeux vidéo disponibles sur le marché serait «violente » (moins de 10\%), celle-ci faisant par ailleurs l'objet d'une signalétique spécifique et de recommandations en fonction de l'âge ( « + 18 ans/adultes ", «+ 16 ans ", ou « 12 ans »). Si problème il y a, cela provient des parents qui doivent surveiller ce qu'ils offrent à leur progéniture... Or, soulignons que ces intervenants parlent là de «l'offre » de jeux vidéo, il est vrai assez diversifiée, mais non des ventes réelles (un jeu comme GTA, déconseillé aux moins de 18 ans en France, se vend mieux que la plupart des «bons » jeux de stratégie dont certains observateurs vantent les mérites, sans parler de «serious games » à la diffusion confidentielle...), ou encore de la pratique réelle de tel ou tel jeu, pouvant passer, chez les adolescents notamment, par l'utilisation de logiciels piratés. En toute connaissance de cause, ils diffusent là encore des informations qui ne sont pas «fausses " au sens strict du terme, mais qui sont statistiquement parlant biaisées.

Les opérations publicitaires ciblant les seniors suscitent un certain nombre de questions. Vise-t-on là, prioritairement, à les amener à s'intéresser à ces produits, dont on leur vante les mérites dans une perspective hygiéniste (entretien du corps et des neurones), et donc à chercher à en convaincre certains d'acheter des jeux pour leur propre usage ? Ou plutôt vise-t-on à les sensibiliser sur la diversité et les bienfaits des jeux vidéo afin qu'ils en offrent à leurs petits-enfants ? Sur ce sujet, il convient d'évoquer une opération orchestrée à Noël 2008 par un fabricant de consoles de jeux. Suite à un partenariat avec un groupe privé de maisons de retraites (représentant moins de $1 \%$ du parc français), celui-ci avait réussi à s'attirer les faveurs des médias, réalisant alors au moment des fêtes des reportages très complaisants vantant les mérites de l'utilisation de cette console auprès du $3^{\mathrm{e}}$ âge et ce, à partir de " témoignages " provenant des quelques établissements où des expériences avaient été réalisées ${ }^{16}$.

S'il reste limité, le « vieillissement » du joueur et les modifications concernant la population des pratiquants de jeux vidéo entraînent concrètement d'autres conséquences devant être interrogées. L'une d'entre elles répond au nom de goldfarming. Les "goldfarmers ", ou " récolteurs d'or ", sont des joueurs qui passent beaucoup de temps à jouer, notamment dans les jeux de rôles en ligne où la progression et le prestige des personnages sont rythmés par la possession d'objets magiques rares pouvant être acquis en échange de ressources collectées dans le cadre du jeu (dont des " pièces d'or » virtuelles). Ils vont monnayer leur temps de jeu auprès d'autres populations, ou, à l'image de mendiants faisant la manche sur les trottoirs, être exploités afin de procurer des revenus à des tiers.

16. On peut notamment se référer à l'émission d'Europe 1, 26 décembre 2008, où nous avions été confronté à un démagogue reproduisant dans son discours les arguments des industriels du jeu vidéo. 
Des cadres ont été amenés à jouer aux jeux vidéo. Ceux-civeulent des produits qui correspondent à leurs attentes : séquences de jeu courtes, permettant ensuite, notamment, de visualiser un classement en ligne (consultable par tous) de ses performances afin de pouvoir se situer par rapport aux autres joueurs. Dans le cadre des jeux de rôles en ligne, par manque de temps, ils délèguent toute une partie du "levelling " (montée de niveaux) du personnage à des tiers contre rémunération, étant prêts à se procurer avec de l'argent réel sur des " marchés parallèles " (en théorie illégaux) ou au sein même du jeu (lorsque cela est autorisé), des biens virtuels permettant de valoriser leur " avatar », voire à racheter à un prix élevé (200 euros par exemple), des « comptes » de personnages puissants ayant atteint un haut-niveau. Cet été, la presse française s'est ainsi émue du témoignage d'un ex-prisonnier chinois, ayant déclaré être obligé lors de son incarcération à jouer 12 heures par jour afin de collecter des ressources pour d'autres joueurs, moyennant finances, et procurer ainsi des revenus à ce centre de détention. Dans son numéro de septembre 2011 ( $\mathrm{n}^{\circ} 64$ ), la revue $M C D$ publiait au sein d'un article évoquant ces phénomènes, une photo censée représenter un " atelier de goldfarming " en Chine, où l'on voyait des teenagers en short, les yeux rivés devant l'écran en train de jouer... Si celle-ci présentait des aspects choquants, elle avait au moins le mérite de poser le problème.

Sans nul doute, des penseurs du siècle dernier aujourd'hui décédés tomberaient des nues s'ils revenaient sur Terre et étaient confrontés à ces logiques. Ils y verraient probablement le stade ultime de l'aliénation, et de l'exploitation, qu'ils pensaient caractéristiques des "sociétés de consommation ». Car la «valeur d'usage » de ces avatars et de ces biens virtuels est singulièrement réduite... A contrario, la façon, disons, "mesurée ", dont certains observateurs rendent compte du phénomène interpelle : finirions-nous par être en quelque sorte « accoutumés » aux logiques sociales problématiques découlant de l'existence de ces loisirs numériques?

\section{Conclusion}

Les travaux que nous conduisons sur la pratique des jeux vidéo depuis plusieurs années se situent clairement dans une perspective de sociologie critique. Telle doit être selon nous la position du sociologue et nous ne pouvons cacher nos craintes sur l'occultation, ou la relativisation, de ce positionnement, au profit de perspectives dites " compréhensives ", voire " pragmatiques », parfois au motif du respect de la "neutralité axiologique », et ce, au sein d'un certain nombre de travaux universitaires produits depuis quelques années sur le champ des pratiques vidéoludiques ${ }^{17}$. En exposant

17. Comme exemples révélateurs de cette tendance, nous pouvons citer S. GENVO, Introduction aux enjeux artistiques et culturels des jeux vidéo, Paris, L’Harmattan, " collection Champs visuels ", 1990 ; H. TER MINASSIAN, S. RUFAT, "Eł si les jeux vidéo servaient à comprendre la géographie ? ", [en ligne], Cybergeo, 2008,http://www.cybergeo.eu/index17502.html. 
quelles sont les logiques développées dans les jeux vidéo les plus pratiqués, et en quelle mesure celles-ci répondent aux attentes d'un "public cible ", en déconstruisant certains artefacts statistiques conçus pour modifier l'image sociale de ces produits, cet article a proposé une synthèse des réflexions que nous avons menées récemment sur la question.

\section{BIBLIOGRAPHIE}

BALZERANI M., « La femme n'est pas l'avenir du jeu vidéo », $M C D, 2011, n^{\circ} 64$, p 72-73.

BEAU FR. (dir.), Culture d'Univers - Jeux en réseau, mondes virtuels, le nouvel âge de la société numérique, Limoges, FYP Editions, 2007, 359 p.

CHAMPAGNE P., Faire l'opinion. Le nouveau jeu politique, Paris, Éditions de Minuit, «collection le sens commun », 1990, $320 \mathrm{p}$.

GENVO S., Introduction aux enjeux artistiques et culturels des jeux vidéo, Paris, L'Harmattan, « collection Champs visuels », 1990, 320 p.

GENVO S., "Les jeux vidéo, un "bien" culturel ?", Médiamorphoses, février 2008, $\mathrm{n}^{\circ} 22,89 \mathrm{p}$.

KLINE ST., DYER-WITHEFORD N., DE PEUTER GR., Digital Play - The interaction of Technology, Culture, and Marketing, Montreal \& Kingston, MC Gill-Queen's University Press, 2003, 376 p.

LIGNON F., « Genre et jeux vidéo », dans BAURENS M., JANNAS F., LIGNON F. (dir.), Le genre en éducation, Grenoble, éditions Eaux Claires, 2007, p. 97-103.

LIGNON F., "L'image de la femme dans les jeux vidéo de combat ", dans ROGER A., TERRET TH. (dir.), Sport et Genre. XIXème-XXème, Paris, L'Harmattan, 2005, vol. 4, p. 171-185.

TER MINASSIAN H., RUFAT S., «Et si les jeux vidéo servaient à comprendre la géographie ? », [en ligne], Cybergeo, 2008, disponibilité et accès http://www.cybergeo.eu/ index17502.html

TRÉMEL L., Jeux de rôles, jeux vidéo, multimédia : les faiseurs de monde, Paris, PUF, « collection sociologie d'aujourd'hui », 2001, 309 p.

TRÉMEL L., « Des jeux vidéo à la "réalité virtuelle" : réflexions sur un objet d'études aux prises avec la réalité sociale » dans TRÉMEL L. (dir.), Les pratiques audiovisuelles. Réflexions sur des questions d'éducation, de culture et de consommation de mass, Dijon, Editions d'un Autre genre, 2009, 249 p.

TRÉMEL L., FORTIN T., Mythologie des jeux vidéo, Paris, Le Cavalier bleu, « collection Mythô », 2009, 95 p. 
Résumé : Basé sur les travaux menés par l'auteur sur la pratique des jeux vidéo depuis une dizaine d'années, l'article se situe dans une perspective de sociologie critique et expose les logiques développées dans les jeux vidéo les plus pratiqués, et comment celles-ci répondent aux attentes d'un « public cible ». En déconstruisant certains artefacts statistiques conçus pour modifier l'image sociale de ces produits, le propos révèle quelles sont les intentions des industriels du loisir et comment ils peuvent " faire l'opinion ".

Mots-clés : jeux vidéo, marketing, lobbying, sondages, sociologie critique.

Abstract : Based on the works led by the author on the practice of the video games since around ten years, the article is situated in a prospect of critical sociology. By explaining what are the logics developed in the most practised video games, and in which measure these meet the expectations of a "target public", by deconstructing certain statistical artefacts conceived to modify the social image of these products, the comment reveals what are the aims of leisure industrialists and how they can "make the opinion".

Keywords : video games, marketing, lobbying, polls, critical sociology. 